\title{
Relativistic corrections to the Sunyaev-Zeldovich effect for extremely hot clusters of galaxies ${ }^{\star}$
}

\author{
N. Itoh ${ }^{1}$ and S. Nozawa ${ }^{2}$ \\ ${ }^{1}$ Department of Physics, Sophia University, 7-1 Kioi-cho, Chiyoda-ku, Tokyo 102-8554, Japan \\ 2 Josai Junior College for Women, 1-1 Keyakidai, Sakado-shi, Saitama 350-0290, Japan \\ e-mail: snozawa@josai.ac.jp \\ Received 28 August 2003 / Accepted 17 November 2003

\begin{abstract}
We present an accurate numerical table for the relativistic corrections to the thermal Sunyaev-Zeldovich effect for clusters of galaxies. The numerical results for the relativistic corrections have been obtained by numerical integration of the collision term of the Boltzmann equation. The numerical table is provided for the ranges $0.002 \leq \theta_{\mathrm{e}} \leq 0.100$ and $0 \leq X \leq 20$, where $\theta_{\mathrm{e}} \equiv k_{\mathrm{B}} T_{\mathrm{e}} / m_{\mathrm{e}} c^{2}, X \equiv \hbar \omega / k_{\mathrm{B}} T_{0}, T_{\mathrm{e}}$ is the electron temperature, $\omega$ is the angular frequency of the photon and $T_{0}$ is the temperature of the cosmic microwave background radiation. We also present an accurate analytic fitting formula that reproduces the numerical results with high precision.
\end{abstract}

Key words. cosmology: cosmic microwave background - cosmology: theory - galaxies: clusters: general radiation mechanisms: thermal - relativity

\section{Introduction}

Compton scattering of the cosmic microwave background (CMB) radiation by hot intracluster gas - the Sunyaev-Zeldovich effect (Zeldovich \& Sunyaev 1969; Sunyaev \& Zeldovich 1972, 1980a,b, 1981) - provides a useful method for studies of cosmology (see recent excellent reviews: Birkinshaw 1999; Carlstrom et al. 2002). The original Sunyaev-Zeldovich formula has been derived from a kinetic equation for the photon distribution function taking into account the Compton scattering by electrons: the Kompaneets equation (Kompaneets 1957; Weymann 1965). The original Kompaneets equation has been derived with a nonrelativistic approximation for the electron. However, recent X-ray observations have revealed the existence of many high-temperature galaxy clusters (David et al. 1993; Arnaud et al. 1994; Markevitch et al. 1994, 1996; Holzapfel et al. 1997; Mushotzky \& Scharf 1997; Markevitch 1998; Allen et al. 2001; Schmidt et al. 2001). In particular, Tucker et al. (1998) reported the discovery of a galaxy cluster with the electron temperature $k_{\mathrm{B}} T_{\mathrm{e}}=17.4 \pm 2.5 \mathrm{keV}$. Wright (1979) and Rephaeli and his collaborator (Rephaeli 1995; Rephaeli \& Yankovitch 1997) have done pioneering work to take into account the relativistic corrections to the Sunyaev-Zeldovich effect for clusters of galaxies.

Send offprint requests to: N. Itoh, e-mail: n_itoh@sophia.ac.jp

* Tables 1-5 are only available in electronic form at the CDS via anonymous ftp to cdsarc.u-strasbg.fr $(130.79 .128 .5)$ or via http://cdsweb.u-strasbg.fr/cgi-bin/qcat?J/A+A/417/827
In recent years remarkable progress has been achieved in the theoretical studies of the relativistic corrections to the Sunyaev-Zeldovich effects for clusters of galaxies. Stebbins (1997) generalized the Kompaneets equation. Itoh et al. (1998) have adopted a relativistically covariant formalism to describe the Compton scattering process (Berestetskii et al. 1982; Buchler \& Yueh 1976), thereby obtaining higher-order relativistic corrections to the thermal Sunyaev-Zeldovich effect in the form of the Fokker-Planck expansion. In their derivation, the scheme to conserve the photon number at every stage of the expansion proposed by Challinor \& Lasenby (1998) played an essential role. The results of Challinor \& Lasenby (1998) are in agreement with those of Itoh et al. (1998). The latter results include higher-order expansions. Itoh et al. (1998) have also calculated the collision integral of the Boltzmann equation numerically and have compared the results with those obtained by the Fokker-Planck expansion method. They have confirmed that the Fokker-Planck expansion method gives an excellent result for $k_{\mathrm{B}} T_{\mathrm{e}} \leq 15 \mathrm{keV}$, where $T_{\mathrm{e}}$ is the electron temperature. For $k_{\mathrm{B}} T_{\mathrm{e}} \geq 15 \mathrm{keV}$, however, the Fokker-Planck expansion results show non-negligible deviations from the results obtained by the numerical integration of the collision term of the Boltzmann equation.

Nozawa et al. (1998b) have extended their method to the case where the galaxy cluster is moving with a peculiar velocity with respect to CMB. They have thereby obtained the relativistic corrections to the kinematical Sunyaev-Zeldovich effect. Challinor \& Lasenby (1999) confirmed the correctness of the result obtained by Nozawa et al. (1998b). Sazonov \& Sunyaev (1998a,b) calculated the kinematical Sunyaev-Zeldovich effect 
by a different method. Their results are in agreement with those of Nozawa et al. (1998b). The latter authors gave the results of the higher-order expansions.

Itoh et al. (2000a) have also applied their method to the calculation of the relativistic corrections to the polarization Sunyaev-Zeldovich effect (Sunyaev \& Zeldovich 1980b, 1981). They thereby confirmed the result of Challinor et al. (1999) which was obtained with a completely different method. Recent work on the polarization Sunyaev-Zeldovich effect include Audit \& Simons (1999), Hansen \& Lilje (1999) and Sazonov \& Sunyaev (1999).

In the present paper we address the numerical calculation of the relativistic corrections to the thermal Sunyaev-Zeldovich effect. As stated above, Itoh et al. (1998) carried out the numerical integration of the collision term of the Boltzmann equation. This method produces exact results without the power series expansion approximation. In view of the discovery of an extremely high temperature galaxy cluster with $k_{\mathrm{B}} T_{\mathrm{e}}=17.4 \pm$ $2.5 \mathrm{keV}$ (Tucker et al. 1998), it would be extremely useful to present the results of the numerical integration of the collision term of the Boltzmann equation. Sazonov \& Sunyaev (1998a,b) reported the results of the Monte Carlo calculations on the relativistic corrections to the Sunyaev-Zeldovich effect. In Sazonov \& Sunyaev (1998b), a numerical table which summarizes the results of the Monte Carlo calculations was presented. This table is of great value when one wishes to calculate the relativistic corrections to the Sunyaev-Zeldovich effect for galaxy clusters of extremely high temperatures. Accurate analytic fitting formulae would be still more convenient for the observers who wish to analyze galaxy clusters with extremely high temperatures. This project has been undertaken by Nozawa et al. (2000). They presented an accurate analyic fitting formula that has a high accuracy for the ranges $0.00 \leq \theta_{\mathrm{e}} \leq 0.05$ and $0 \leq X \leq 20$, where $\theta_{\mathrm{e}} \equiv k_{\mathrm{B}} T_{\mathrm{e}} / m_{\mathrm{e}} c^{2}, X \equiv \hbar \omega / k_{\mathrm{B}} T_{0}$. Another fitting formula with a still higher precision that is valid for the more limited ranges $0.00 \leq \theta_{\mathrm{e}} \leq 0.035,0 \leq X \leq 15$ was developed (Itoh et al. 2002b). Relativistic corrections to the double scattering effect on the Sunyaev-Zeldovich effect was calculated by Itoh et al. (2001). They presented the Fokker-Planck expansion results up to the $\theta_{\mathrm{e}}^{6}$ correction term as well as the exact numerical results obtained by the numerical integration of the Boltzmann collision term. Dolgov et al. (2001) carried out an independent numerical calculation. Their result shows an excellent agreement with that of Itoh et al. (2001) for the case of small optical depth.

It now appears that all the necessary theoretical tools are ready for the accurate analysis of the observational data of the Sunyaev-Zeldovich effect for clusters of galaxies. However, we notice that the numerical data of Sazonov \& Sunyaev (1998b) and the fitting formula of Nozawa et al. (2000) are both valid for the range $\theta_{\mathrm{e}} \leq 0.05$. Very recently Kitayama et al. (2003) measured the Sunyaev-Zeldovich effect in the galaxy cluster RX J1347-1145. They have discovered that the south-east excess component of this galaxy cluster has a temperature $k_{\mathrm{B}} T_{\mathrm{ex}}=28.5 \pm 7.3 \mathrm{keV}$. They attributed the high temperature of this excess component to a recent major merger as discussed by Sarazin (2003). Therefore it is important to present accurate numerical data for $k_{\mathrm{B}} T_{\mathrm{e}} \geq 25 \mathrm{keV}$. This is the aim of this paper. The following discussion is valid not only for the entire isothermal galaxy cluster but also for a part of it. When the galaxy cluster has a temperature distribution, one only needs to integrate along the line of sight. It is not very likely, however, that a galaxy cluster exists whose component has a temperature as high as $50 \mathrm{keV}$. Nevertheless, compilation of the results for $k_{\mathrm{B}} T_{\mathrm{e}}=50 \mathrm{keV}$ will be useful when one discusses the nonthermal Sunyaev-Zeldovich effect corresponding to this energy range. Therefore we will cover a very wide temperature range $0.002 \leq \theta_{\mathrm{e}} \leq 0.100$, where $\theta_{\mathrm{e}} \equiv k_{\mathrm{B}} T_{\mathrm{e}} / m_{\mathrm{e}} c^{2}$. For the analyses of the galaxy clusters with extremely high temperatures, the results of the calculation of the relativistic thermal bremsstrahlung Gaunt factor (Nozawa et al. 1998a) and their accurate analytic fitting formulae (Itoh et al. 2000b) will be useful. The nonrelativistic electron-electron thermal bremsstrahlung Gaunt factor has been also calculated by Itoh et al. (2002a).

The present paper is organized as follows. In Sect. 2 we give the method of the calculation. In Sect. 3 we give the numerical table. In Sect. 4 we give the analytic fitting formula. Concluding remarks will be given in Sect. 5 .

\section{Boltzmann equation}

We will formulate the kinetic equation for the photon distribution function using a relativistically covariant formalism (Berestetskii et al. 1982; Buchler \& Yueh 1976). As a reference system, we choose the system that is fixed to the center of mass of the cluster of galaxies. This choice of the reference system allows us to carry out all the calculations in the most straightforward way. We will use the invariant amplitude for the Compton scattering as given by Berestetskii et al. (1982) and by Buchler \& Yueh (1976).

The time evolution of the photon distribution function $n(\omega)$ is written as

$$
\begin{aligned}
& \frac{\partial n(\omega)}{\partial t}=-2 \int \frac{\mathrm{d}^{3} p}{(2 \pi)^{3}} \mathrm{~d}^{3} p^{\prime} \mathrm{d}^{3} k^{\prime} W\left\{n(\omega)\left[1+n\left(\omega^{\prime}\right)\right] f(E)\right. \\
& \left.\quad-n\left(\omega^{\prime}\right)[1+n(\omega)] f\left(E^{\prime}\right)\right\} \\
& W=\frac{\left(\mathrm{e}^{2} / 4 \pi\right)^{2} \bar{X} \delta^{4}\left(p+k-p^{\prime}-k^{\prime}\right)}{2 \omega \omega^{\prime} E E^{\prime}} \\
& \bar{X}=-\left(\frac{\kappa}{\kappa^{\prime}}+\frac{\kappa^{\prime}}{\kappa}\right)+4 m^{4}\left(\frac{1}{\kappa}+\frac{1}{\kappa^{\prime}}\right)^{2}-4 m^{2}\left(\frac{1}{\kappa}+\frac{1}{\kappa^{\prime}}\right) \\
& \kappa=-2(p \cdot k)=-2 \omega E\left(1-\frac{|\boldsymbol{p}|}{E} \cos \alpha\right) \\
& \kappa^{\prime}=2\left(p \cdot k^{\prime}\right)=2 \omega^{\prime} E\left(1-\frac{|\boldsymbol{p}|}{E} \cos \alpha^{\prime}\right) .
\end{aligned}
$$

In the above $W$ is the transition probability corresponding to the Compton scattering. The four-momenta of the initial electron and photon are $p=(E, \boldsymbol{p})$ and $k=(\omega, \boldsymbol{k})$, respectively. The four-momenta of the final electron and photon are $p^{\prime}=\left(E^{\prime}, \boldsymbol{p}^{\prime}\right)$ and $k^{\prime}=\left(\omega^{\prime}, \boldsymbol{k}^{\prime}\right)$, respectively. The angles $\alpha$ and $\alpha^{\prime}$ are the angles between $\boldsymbol{p}$ and $\boldsymbol{k}$, and between $\boldsymbol{p}$ and $\boldsymbol{k}^{\prime}$, respectively. Throughout this paper, we use the natural unit $\hbar=c=1$ unit, unless otherwise stated explicitly. 
Table 1. Numerical values of $\Delta I / y$ for $\theta_{\mathrm{e}}=0.002-0.020$. The full content is available at the CDS.

\begin{tabular}{rrrrrrrrrrrr}
\hline \hline & $\theta_{\mathrm{e}}=0.002$ & $\theta_{\mathrm{e}}=0.004$ & $\theta_{\mathrm{e}}=0.006$ & $\theta_{\mathrm{e}}=0.008$ & $\theta_{\mathrm{e}}=0.010$ & $\theta_{\mathrm{e}}=0.012$ & $\theta_{\mathrm{e}}=0.014$ & $\theta_{\mathrm{e}}=0.016$ & $\theta_{\mathrm{e}}=0.018$ & $\theta_{\mathrm{e}}=0.020$ \\
\hline$X=0.0$ & $0.000 \mathrm{E}+0$ & $0.000 \mathrm{E}+0$ & $0.000 \mathrm{E}+0$ & $0.000 \mathrm{E}+0$ & $0.000 \mathrm{E}+0$ & $0.000 \mathrm{E}+0$ & $0.000 \mathrm{E}+0$ & $0.000 \mathrm{E}+0$ & $0.000 \mathrm{E}+0$ & $0.000 \mathrm{E}+0$ \\
$X=0.2$ & $-1.584 \mathrm{E}-4$ & $-3.157 \mathrm{E}-4$ & $-4.719 \mathrm{E}-4$ & $-6.271 \mathrm{E}-4$ & $-7.812 \mathrm{E}-4$ & $-9.343 \mathrm{E}-4$ & $-1.086 \mathrm{E}-3$ & $-1.237 \mathrm{E}-3$ & $-1.387 \mathrm{E}-3$ & $-1.536 \mathrm{E}-3$ \\
$X=0.4$ & $-6.209 \mathrm{E}-4$ & $-1.237 \mathrm{E}-3$ & $-1.849 \mathrm{E}-3$ & $-2.457 \mathrm{E}-3$ & $-3.060 \mathrm{E}-3$ & $-3.659 \mathrm{E}-3$ & $-4.254 \mathrm{E}-3$ & $-4.844 \mathrm{E}-3$ & $-5.431 \mathrm{E}-3$ & $-6.013 \mathrm{E}-3$ \\
\hline
\end{tabular}

By ignoring the degeneracy effects, we have the relativistic Maxwellian distribution for electrons with temperature $T_{\mathrm{e}}$ as follows

$$
\begin{aligned}
f(E) & =\left[\mathrm{e}^{[(E-m)-(\mu-m)] / k_{\mathrm{B}} T_{\mathrm{e}}}+1\right]^{-1} \\
& \approx \mathrm{e}^{-[K-(\mu-m)] / k_{\mathrm{B}} T_{\mathrm{e}}},
\end{aligned}
$$

where $K \equiv(E-m)$ is the kinetic energy of the initial electron, and $(\mu-m)$ is the non-relativistic chemical potential of the electron. We now introduce the quantities

$x \equiv \frac{\omega}{k_{\mathrm{B}} T_{\mathrm{e}}}$,

$\Delta x \equiv \frac{\omega^{\prime}-\omega}{k_{\mathrm{B}} T_{\mathrm{e}}}$.

Substituting Eqs. (2.6)-(2.8) into Eq. (2.1), we obtain

$$
\begin{aligned}
\frac{\partial n(\omega)}{\partial t}= & -2 \int \frac{\mathrm{d}^{3} p}{(2 \pi)^{3}} \mathrm{~d}^{3} p^{\prime} \mathrm{d}^{3} k^{\prime} W f(E)\left\{\left[1+n\left(\omega^{\prime}\right)\right] n(\omega)\right. \\
& \left.-[1+n(\omega)] n\left(\omega^{\prime}\right) \mathrm{e}^{\Delta x}\right\} .
\end{aligned}
$$

Equation (2.9) is our basic equation. We will denote the Thomson scattering cross section by $\sigma_{T}$, and the electron number density by $N_{\mathrm{e}}$. We will define

$\theta_{\mathrm{e}} \equiv \frac{k_{\mathrm{B}} T_{\mathrm{e}}}{m_{\mathrm{e}} c^{2}}$

$y \equiv \sigma_{T} \int \mathrm{d} \ell N_{\mathrm{e}}$,

where $T_{\mathrm{e}}$ is the electron temperature, and the integral in Eq. (2.11) is over the path length of the galaxy cluster. The reader should notice that our definition (2.11) is different from the usual notation. By introducing the initial photon distribution of the $\mathrm{CMB}$ radiation which is assumed to be Planckian with temperature $T_{0}$

$n_{0}(X)=\frac{1}{\mathrm{e}^{X}-1}$,

$X \equiv \frac{\omega}{k_{\mathrm{B}} T_{0}}$,

we rewrite Eq. (2.9) as

$$
\frac{\Delta n(X)}{n_{0}(X)}=y F\left(\theta_{\mathrm{e}}, X\right)
$$

We obtain the function $F\left(\theta_{\mathrm{e}}, X\right)$ by numerical integration of the collision term of the Boltzmann Eq. (2.9). The accuracy of the

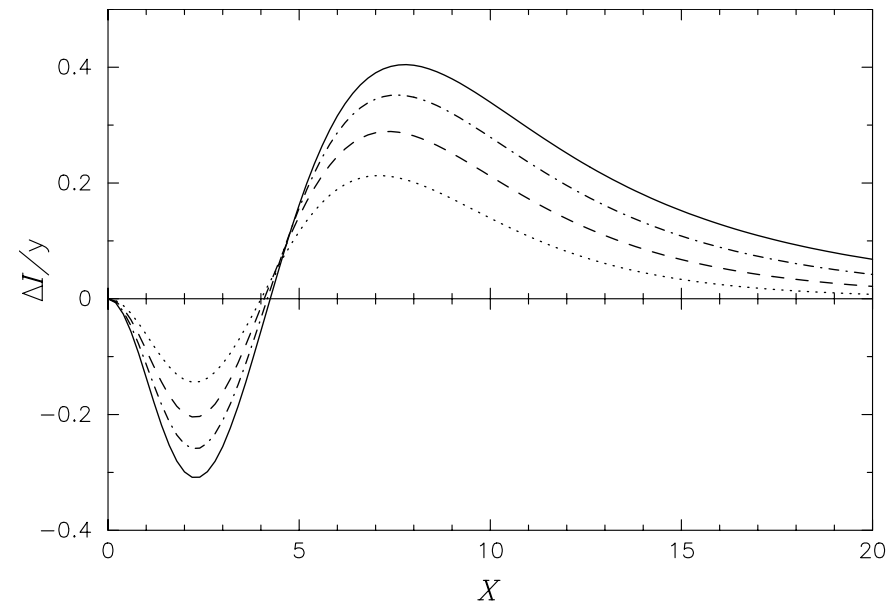

Fig. 1. Spectral intensity distortion $\Delta I / y$ as a function of $X$. The dotted, dashed, dash-dotted, and solid curves correspond to the cases for $\theta_{\mathrm{e}}=0.04,0.06,0.08,0.10$, respectively.

numerical integration is about $10^{-5}$. We confirm that the condition of the photon number conservation

$\int \mathrm{d} X X^{2} \Delta n(X)=0$

is satisfied with an accuracy better than $10^{-9}$.

We define the distortion of the spectral intensity as

$$
\begin{aligned}
\Delta I & \equiv \frac{X^{3}}{\mathrm{e}^{X}-1} \frac{\Delta n(X)}{n_{0}(X)} \\
& =y \frac{X^{3}}{\mathrm{e}^{X}-1} F\left(\theta_{\mathrm{e}}, X\right) .
\end{aligned}
$$

The graph of $\Delta I / y$ is shown in Fig. 1.

\section{Numerical results}

In Tables 1-5 we give the numerical results of the function

$$
\begin{aligned}
\frac{\Delta I}{y} & =\frac{1}{y} \frac{X^{3}}{\mathrm{e}^{X}-1} \frac{\Delta n(X)}{n_{0}(X)} \\
& =\frac{X^{3}}{\mathrm{e}^{X}-1} F\left(\theta_{\mathrm{e}}, X\right) .
\end{aligned}
$$

In Table 1 we show the numerical values of the function $\Delta I / y$ for $\theta_{\mathrm{e}}=0.002-0.020,0 \leq X \leq 20$. Tables $2-5$ are the same as Table 1 but for $\theta_{\mathrm{e}}=0.022-0.040, \theta_{\mathrm{e}}=0.042-0.060, \theta_{\mathrm{e}}=$ $0.062-0.080, \theta_{\mathrm{e}}=0.082-0.100$, respectively. The size of these tables are large. We show the first lines of these tables in the present paper. The full content of these tables is available in electronic form at the CDS. 
Table 2. Numerical values of $\Delta I / y$ for $\theta_{\mathrm{e}}=0.022-0.040$. The full content is available at the CDS.

\begin{tabular}{rrrrrrrrrrrr}
\hline \hline & $\theta_{\mathrm{e}}=0.022$ & $\theta_{\mathrm{e}}=0.024$ & $\theta_{\mathrm{e}}=0.026$ & $\theta_{\mathrm{e}}=0.028$ & $\theta_{\mathrm{e}}=0.030$ & $\theta_{\mathrm{e}}=0.032$ & $\theta_{\mathrm{e}}=0.034$ & $\theta_{\mathrm{e}}=0.036$ & $\theta_{\mathrm{e}}=0.038$ & $\theta_{\mathrm{e}}=0.040$ \\
\hline$X=0.0$ & $0.000 \mathrm{E}+0$ & $0.000 \mathrm{E}+0$ & $0.000 \mathrm{E}+0$ & $0.000 \mathrm{E}+0$ & $0.000 \mathrm{E}+0$ & $0.000 \mathrm{E}+0$ & $0.000 \mathrm{E}+0$ & $0.000 \mathrm{E}+0$ & $0.000 \mathrm{E}+0$ & $0.000 \mathrm{E}+0$ \\
$X=0.2$ & $-1.684 \mathrm{E}-3$ & $-1.831 \mathrm{E}-3$ & $-1.977 \mathrm{E}-3$ & $-2.122 \mathrm{E}-3$ & $-2.267 \mathrm{E}-3$ & $-2.410 \mathrm{E}-3$ & $-2.552 \mathrm{E}-3$ & $-2.694 \mathrm{E}-3$ & $-2.834 \mathrm{E}-3$ & $-2.973 \mathrm{E}-3$ \\
$X=0.4$ & $-6.591 \mathrm{E}-3$ & $-7.166 \mathrm{E}-3$ & $-7.736 \mathrm{E}-3$ & $-8.302 \mathrm{E}-3$ & $-8.864 \mathrm{E}-3$ & $-9.423 \mathrm{E}-3$ & $-9.977 \mathrm{E}-3$ & $-1.053 \mathrm{E}-2$ & $-1.107 \mathrm{E}-2$ & $-1.162 \mathrm{E}-2$ \\
\hline
\end{tabular}

Table 3. Numerical values of $\Delta I / y$ for $\theta_{\mathrm{e}}=0.042-0.060$. The full content is available at the CDS.

\begin{tabular}{rrrrrrrrrrrr}
\hline \hline & $\theta_{\mathrm{e}}=0.042$ & $\theta_{\mathrm{e}}=0.044$ & $\theta_{\mathrm{e}}=0.046$ & $\theta_{\mathrm{e}}=0.048$ & $\theta_{\mathrm{e}}=0.050$ & $\theta_{\mathrm{e}}=0.052$ & $\theta_{\mathrm{e}}=0.054$ & $\theta_{\mathrm{e}}=0.056$ & $\theta_{\mathrm{e}}=0.058$ & $\theta_{\mathrm{e}}=0.060$ \\
\hline$X=0.0$ & $0.000 \mathrm{E}+0$ & $0.000 \mathrm{E}+0$ & $0.000 \mathrm{E}+0$ & $0.000 \mathrm{E}+0$ & $0.000 \mathrm{E}+0$ & $0.000 \mathrm{E}+0$ & $0.000 \mathrm{E}+0$ & $0.000 \mathrm{E}+0$ & $0.000 \mathrm{E}+0$ & $0.000 \mathrm{E}+0$ \\
$X=0.2$ & $-3.112 \mathrm{E}-3$ & $-3.250 \mathrm{E}-3$ & $-3.387 \mathrm{E}-3$ & $-3.523 \mathrm{E}-3$ & $-3.658 \mathrm{E}-3$ & $-3.792 \mathrm{E}-3$ & $-3.925 \mathrm{E}-3$ & $-4.058 \mathrm{E}-3$ & $-4.190 \mathrm{E}-3$ & $-4.320 \mathrm{E}-3$ \\
$X=0.4$ & $-1.216 \mathrm{E}-2$ & $-1.269 \mathrm{E}-2$ & $-1.323 \mathrm{E}-2$ & $-1.375 \mathrm{E}-2$ & $-1.428 \mathrm{E}-2$ & $-1.480 \mathrm{E}-2$ & $-1.532 \mathrm{E}-2$ & $-1.583 \mathrm{E}-2$ & $-1.634 \mathrm{E}-2$ & $-1.685 \mathrm{E}-2$ \\
\hline
\end{tabular}

Table 4. Numerical values of $\Delta I / y$ for $\theta_{\mathrm{e}}=0.062-0.080$. The full content is available at the CDS.

\begin{tabular}{rrrrrrrrrrrr}
\hline \hline & $\theta_{\mathrm{e}}=0.062$ & $\theta_{\mathrm{e}}=0.064$ & $\theta_{\mathrm{e}}=0.066$ & $\theta_{\mathrm{e}}=0.068$ & $\theta_{\mathrm{e}}=0.070$ & $\theta_{\mathrm{e}}=0.072$ & $\theta_{\mathrm{e}}=0.074$ & $\theta_{\mathrm{e}}=0.076$ & $\theta_{\mathrm{e}}=0.078$ & $\theta_{\mathrm{e}}=0.080$ \\
\hline$X=0.0$ & $0.000 \mathrm{E}+0$ & $0.000 \mathrm{E}+0$ & $0.000 \mathrm{E}+0$ & $0.000 \mathrm{E}+0$ & $0.000 \mathrm{E}+0$ & $0.000 \mathrm{E}+0$ & $0.000 \mathrm{E}+0$ & $0.000 \mathrm{E}+0$ & $0.000 \mathrm{E}+0$ & $0.000 \mathrm{E}+0$ \\
$X=0.2$ & $-4.451 \mathrm{E}-3$ & $-4.580 \mathrm{E}-3$ & $-4.708 \mathrm{E}-3$ & $-4.836 \mathrm{E}-3$ & $-4.963 \mathrm{E}-3$ & $-5.089 \mathrm{E}-3$ & $-5.214 \mathrm{E}-3$ & $-5.339 \mathrm{E}-3$ & $-5.463 \mathrm{E}-3$ & $-5.586 \mathrm{E}-3$ \\
$X=0.4$ & $-1.735 \mathrm{E}-2$ & $-1.786 \mathrm{E}-2$ & $-1.835 \mathrm{E}-2$ & $-1.885 \mathrm{E}-2$ & $-1.934 \mathrm{E}-2$ & $-1.983 \mathrm{E}-2$ & $-2.031 \mathrm{E}-2$ & $-2.079 \mathrm{E}-2$ & $-2.127 \mathrm{E}-2$ & $-2.174 \mathrm{E}-2$ \\
\hline
\end{tabular}

Table 5. Numerical values of $\Delta I / y$ for $\theta_{\mathrm{e}}=0.082-0.100$. The full content is available at the CDS.

\begin{tabular}{rrrrrrrrrrrr}
\hline \hline & $\theta_{\mathrm{e}}=0.082$ & $\theta_{\mathrm{e}}=0.084$ & $\theta_{\mathrm{e}}=0.086$ & $\theta_{\mathrm{e}}=0.088$ & $\theta_{\mathrm{e}}=0.090$ & $\theta_{\mathrm{e}}=0.092$ & $\theta_{\mathrm{e}}=0.094$ & $\theta_{\mathrm{e}}=0.096$ & $\theta_{\mathrm{e}}=0.098$ & $\theta_{\mathrm{e}}=0.100$ \\
\hline$X=0.0$ & $0.000 \mathrm{E}+0$ & $0.000 \mathrm{E}+0$ & $0.000 \mathrm{E}+0$ & $0.000 \mathrm{E}+0$ & $0.000 \mathrm{E}+0$ & $0.000 \mathrm{E}+0$ & $0.000 \mathrm{E}+0$ & $0.000 \mathrm{E}+0$ & $0.000 \mathrm{E}+0$ & $0.000 \mathrm{E}+0$ \\
$X=0.2$ & $-5.708 \mathrm{E}-3$ & $-5.829 \mathrm{E}-3$ & $-5.950 \mathrm{E}-3$ & $-6.070 \mathrm{E}-3$ & $-6.189 \mathrm{E}-3$ & $-6.308 \mathrm{E}-3$ & $-6.426 \mathrm{E}-3$ & $-6.543 \mathrm{E}-3$ & $-6.659 \mathrm{E}-3$ & $-6.775 \mathrm{E}-3$ \\
$X=0.4$ & $-2.222 \mathrm{E}-2$ & $-2.269 \mathrm{E}-2$ & $-2.315 \mathrm{E}-2$ & $-2.361 \mathrm{E}-2$ & $-2.407 \mathrm{E}-2$ & $-2.453 \mathrm{E}-2$ & $-2.498 \mathrm{E}-2$ & $-2.543 \mathrm{E}-2$ & $-2.588 \mathrm{E}-2$ & $-2.633 \mathrm{E}-2$ \\
\hline
\end{tabular}

\section{Analytic fitting formula}

We express the analytic fitting formula for $0.05 \leq \theta_{\mathrm{e}} \leq 0.10$, $0 \leq X \leq 17$ in the following way.

(i) For $0 \leq X \leq 1.2$

$$
\begin{aligned}
\frac{\Delta I}{y} & =\frac{1}{y} \frac{X^{3}}{\mathrm{e}^{X}-1} \frac{\Delta n(X)}{n_{0}(X)} \\
& =\frac{\theta_{\mathrm{e}} X^{4} \mathrm{e}^{X}}{\left(\mathrm{e}^{X}-1\right)^{2}}\left(Y_{0}+\theta_{\mathrm{e}} Y_{1}+\theta_{\mathrm{e}}^{2} Y_{2}+\theta_{\mathrm{e}}^{3} Y_{3}+\theta_{\mathrm{e}}^{4} Y_{4}\right) .
\end{aligned}
$$

The explicit expressions for $Y_{0}, Y_{1}, Y_{2}, Y_{3}$, and $Y_{4}$ have been presented in Itoh et al. (1998).

(ii) For $1.2 \leq X \leq 17$

$$
\frac{\Delta I}{y}=\theta_{\mathrm{e}} X^{2} \mathrm{e}^{-X}\left(X-X_{0}\right) G\left(\theta_{\mathrm{e}}, X\right)
$$

In Eq. (4.2) $X_{0}$ is the crossover frequency, where the thermal Sunyaev-Zeldovich effect vanishes. We use the following expression for $X_{0}$ obtained by Itoh et al. (1998):

$$
X_{0}=3.830\left(1+1.1674 \theta_{\mathrm{e}}-0.8533 \theta_{\mathrm{e}}^{2}\right) \text {. }
$$

The explicit form of the analytic fitting function $G\left(\theta_{\mathrm{e}}, X\right)$ is given as follows:

$G\left(\theta_{\mathrm{e}}, X\right)=\sum_{i, j=0}^{12} a_{i j} \Theta_{\mathrm{e}}^{i} Z^{j}$,

$\Theta_{\mathrm{e}} \equiv 10 \theta_{\mathrm{e}}, \quad 0.05 \leq \theta_{\mathrm{e}} \leq 0.100$,

$Z \equiv \frac{1}{20} X, \quad 1.2 \leq X \leq 17$.

The coefficients $a_{i j}$ are presented in Table 6 . In order to analyze the accuracy of the fitting formula, we define (Hansen 2003)

$Q \equiv \frac{\frac{\Delta I_{\text {fit }}}{y}-\frac{\Delta I_{\text {num }}}{y}}{\left|\frac{\Delta I_{\text {num }}}{y}\right|+\left|\frac{\Delta I_{\text {num }}}{y}-\frac{\theta_{\mathrm{e}} X^{4} \mathrm{e}^{X}}{\left(\mathrm{e}^{X}-1\right)^{2}} Y_{0}\right|}$,

where $\Delta I_{\text {num }}$ and $\Delta I_{\text {fit }}$ are the numerical calculation and the fitting result for the distortion of the spectral intensity, respectively. The accuracy of the fitting formula is generally better than $0.2 \%$. The accuracy of the presented fitting formula is shown in Fig. 2. 
Table 6. Coefficients $a_{i j}$.

\begin{tabular}{rrrrrrrr}
\hline \hline$i$ & \multicolumn{1}{c}{$j=0$} & $j=1$ & $j=2$ & $j=3$ & $j=4$ & $j=5$ & $j=6$ \\
\hline 0 & $-1.81317 \mathrm{E}+1$ & $9.97038 \mathrm{E}+1$ & $-6.07438 \mathrm{E}+1$ & $1.05143 \mathrm{E}+3$ & $-2.86734 \mathrm{E}+3$ & $7.73353 \mathrm{E}+3$ & $-8.16644 \mathrm{E}+3$ \\
1 & $1.68733 \mathrm{E}+2$ & $-6.07829 \mathrm{E}+2$ & $1.14933 \mathrm{E}+3$ & $-2.42382 \mathrm{E}+2$ & $-7.73030 \mathrm{E}+2$ & $5.33993 \mathrm{E}+3$ & $-4.03443 \mathrm{E}+3$ \\
2 & $-6.69883 \mathrm{E}+2$ & $1.59654 \mathrm{E}+3$ & $-3.33375 \mathrm{E}+3$ & $-2.13234 \mathrm{E}+3$ & $-1.80812 \mathrm{E}+2$ & $3.75605 \mathrm{E}+3$ & $-4.75180 \mathrm{E}+3$ \\
3 & $1.56222 \mathrm{E}+3$ & $-1.78598 \mathrm{E}+3$ & $5.13747 \mathrm{E}+3$ & $4.10404 \mathrm{E}+3$ & $5.54775 \mathrm{E}+2$ & $-3.89994 \mathrm{E}+3$ & $-1.22455 \mathrm{E}+3$ \\
4 & $-2.34712 \mathrm{E}+3$ & $2.78197 \mathrm{E}+2$ & $-5.49648 \mathrm{E}+3$ & $-5.94988 \mathrm{E}+2$ & $-1.47060 \mathrm{E}+3$ & $-2.84032 \mathrm{E}+2$ & $-1.15352 \mathrm{E}+3$ \\
5 & $1.92894 \mathrm{E}+3$ & $1.17970 \mathrm{E}+3$ & $3.13650 \mathrm{E}+3$ & $-2.91121 \mathrm{E}+2$ & $-1.15006 \mathrm{E}+3$ & $4.17375 \mathrm{E}+3$ & $-3.31788 \mathrm{E}+2$ \\
6 & $6.40881 \mathrm{E}+2$ & $-6.81789 \mathrm{E}+2$ & $1.20037 \mathrm{E}+3$ & $-3.27298 \mathrm{E}+3$ & $1.02988 \mathrm{E}+2$ & $2.03514 \mathrm{E}+3$ & $-2.80502 \mathrm{E}+3$ \\
7 & $-4.02494 \mathrm{E}+3$ & $-1.37983 \mathrm{E}+3$ & $-1.65623 \mathrm{E}+3$ & $7.36120 \mathrm{E}+1$ & $2.66656 \mathrm{E}+3$ & $-2.30516 \mathrm{E}+3$ & $5.22182 \mathrm{E}+3$ \\
8 & $4.59247 \mathrm{E}+3$ & $3.04203 \mathrm{E}+3$ & $-2.11039 \mathrm{E}+3$ & $1.32383 \mathrm{E}+3$ & $1.10646 \mathrm{E}+3$ & $-3.53827 \mathrm{E}+3$ & $-1.12073 \mathrm{E}+3$ \\
9 & $-1.61848 \mathrm{E}+3$ & $-1.83704 \mathrm{E}+3$ & $2.06738 \mathrm{E}+3$ & $4.00292 \mathrm{E}+3$ & $-3.72824 \mathrm{E}+1$ & $9.10086 \mathrm{E}+2$ & $3.72526 \mathrm{E}+3$ \\
10 & $-1.00239 \mathrm{E}+3$ & $-1.24281 \mathrm{E}+3$ & $2.46998 \mathrm{E}+3$ & $-4.25837 \mathrm{E}+3$ & $-1.83515 \mathrm{E}+2$ & $-6.47138 \mathrm{E}+2$ & $-7.35806 \mathrm{E}+3$ \\
11 & $1.04911 \mathrm{E}+3$ & $2.07475 \mathrm{E}+3$ & $-3.83953 \mathrm{E}+3$ & $7.79924 \mathrm{E}+2$ & $-4.08658 \mathrm{E}+3$ & $4.43432 \mathrm{E}+3$ & $3.23015 \mathrm{E}+2$ \\
12 & $-2.61041 \mathrm{E}+2$ & $-7.22803 \mathrm{E}+2$ & $1.34581 \mathrm{E}+3$ & $5.90851 \mathrm{E}+2$ & $3.32198 \mathrm{E}+2$ & $2.58340 \mathrm{E}+3$ & $-5.97604 \mathrm{E}+2$ \\
\hline
\end{tabular}

\begin{tabular}{rrrcccr}
\hline \hline$i$ & $j=7$ & $j=8$ & $j=9$ & $j=10$ & $j=11$ & $j=12$ \\
\hline 0 & $-5.37712 \mathrm{E}+3$ & $1.52226 \mathrm{E}+4$ & $7.18726 \mathrm{E}+3$ & $-1.39548 \mathrm{E}+4$ & $-2.08464 \mathrm{E}+4$ & $1.79040 \mathrm{E}+4$ \\
1 & $3.00692 \mathrm{E}+3$ & $9.58809 \mathrm{E}+3$ & $8.16574 \mathrm{E}+3$ & $-6.13322 \mathrm{E}+3$ & $-1.48117 \mathrm{E}+4$ & $3.43816 \mathrm{E}+4$ \\
2 & $-4.50495 \mathrm{E}+3$ & $5.38753 \mathrm{E}+3$ & $5.03355 \mathrm{E}+3$ & $-1.18396 \mathrm{E}+4$ & $-8.58473 \mathrm{E}+3$ & $3.96316 \mathrm{E}+4$ \\
3 & $1.03747 \mathrm{E}+3$ & $4.32237 \mathrm{E}+3$ & $1.03805 \mathrm{E}+3$ & $-1.47172 \mathrm{E}+4$ & $-1.23591 \mathrm{E}+4$ & $1.77290 \mathrm{E}+4$ \\
4 & $-1.17893 \mathrm{E}+3$ & $7.01209 \mathrm{E}+3$ & $4.75631 \mathrm{E}+3$ & $-5.13807 \mathrm{E}+3$ & $-8.73615 \mathrm{E}+3$ & $9.41580 \mathrm{E}+3$ \\
5 & $1.37973 \mathrm{E}+3$ & $-2.48966 \mathrm{E}+3$ & $4.82005 \mathrm{E}+3$ & $-1.06121 \mathrm{E}+4$ & $-1.19394 \mathrm{E}+4$ & $1.34908 \mathrm{E}+4$ \\
6 & $8.83880 \mathrm{E}+2$ & $1.68409 \mathrm{E}+3$ & $4.26227 \mathrm{E}+3$ & $-6.37868 \mathrm{E}+3$ & $-1.11597 \mathrm{E}+4$ & $1.46861 \mathrm{E}+4$ \\
7 & $-8.53317 \mathrm{E}+3$ & $3.75800 \mathrm{E}+2$ & $8.49249 \mathrm{E}+2$ & $-6.88736 \mathrm{E}+3$ & $-1.01475 \mathrm{E}+4$ & $4.75820 \mathrm{E}+3$ \\
8 & $-5.47633 \mathrm{E}+3$ & $9.85745 \mathrm{E}+3$ & $5.72138 \mathrm{E}+3$ & $6.86444 \mathrm{E}+3$ & $-5.72696 \mathrm{E}+3$ & $1.29053 \mathrm{E}+3$ \\
9 & $3.41895 \mathrm{E}+3$ & $1.31241 \mathrm{E}+3$ & $6.68089 \mathrm{E}+3$ & $-4.34269 \mathrm{E}+3$ & $-5.42296 \mathrm{E}+3$ & $2.83445 \mathrm{E}+3$ \\
10 & $-1.50866 \mathrm{E}+3$ & $-2.47275 \mathrm{E}+3$ & $9.09399 \mathrm{E}+3$ & $-2.75851 \mathrm{E}+3$ & $-6.75104 \mathrm{E}+3$ & $7.00899 \mathrm{E}+2$ \\
11 & $6.16180 \mathrm{E}+3$ & $-1.00851 \mathrm{E}+4$ & $7.65063 \mathrm{E}+3$ & $1.52880 \mathrm{E}+3$ & $-6.08330 \mathrm{E}+3$ & $1.23369 \mathrm{E}+3$ \\
12 & $-4.34018 \mathrm{E}+3$ & $-3.58925 \mathrm{E}+3$ & $2.59165 \mathrm{E}+3$ & $6.76140 \mathrm{E}+3$ & $-6.22138 \mathrm{E}+3$ & $4.40668 \mathrm{E}+3$ \\
\hline
\end{tabular}

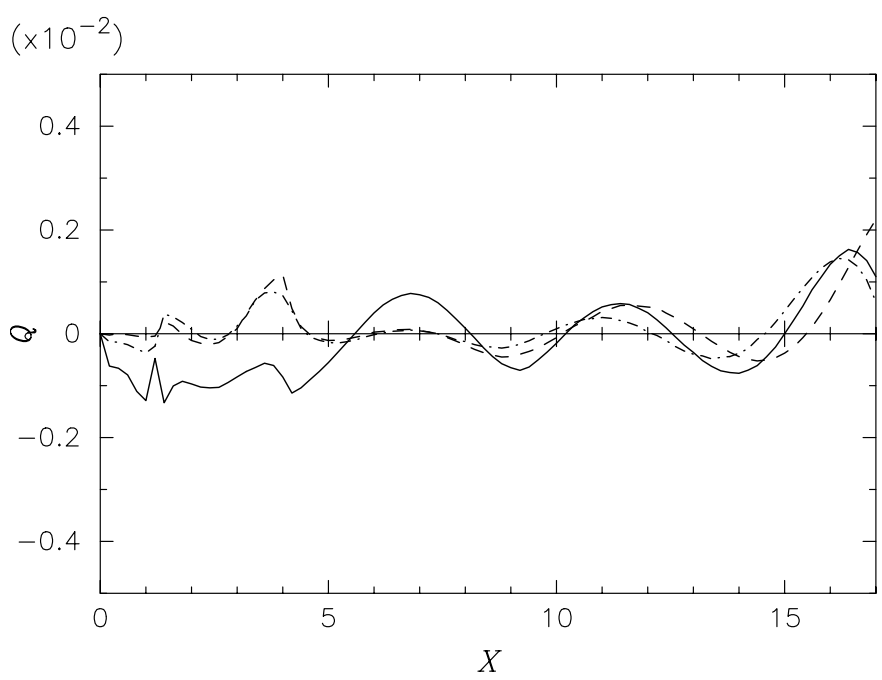

Fig. 2. The accuracy of the present fitting formula for various values of $\theta_{\mathrm{e}}$. The dashed, dash-dotted, and solid curves correspond to $\theta_{\mathrm{e}}=$ $0.050,0.075$, and 0.100 , respectively.

\section{Concluding remarks}

We have calculated the relativistic corrections to the thermal Sunyaev-Zeldovich effect for clusters of galaxies by numerical integration of the collision term of the Boltzmann equation. The emphasis is placed on the cases of high electron temperatures to provide the theoretical tools for the analysis of extremely high-temperature galaxy cluster substructures with $k_{\mathrm{B}} T_{\mathrm{e}} \geq 25 \mathrm{keV}$ that are likely to be discovered by the ongoing X-ray and Sunyaev-Zeldovich effect observations. We have presented a detailed numerical table and an accurate analytic fitting formula. We will be most pleased to provide the computer subroutines for the numerical table with a standard interpolation package as well as for the analytic fitting formula if requested.

Acknowledgements. We thank Y. Oyanagi for allowing us to use the least square fitting program SALS. We also thank Y. Suto and T. Kitayama for discussions on their observational results that have inspired us to write this paper. It is also a pleasure to thank Y. Rephaeli for the discussion on the calculation of the thermal Sunyaev-Zeldovich effect for high-temperature galaxy clusters. We also thank our referee for many valuable comments that have greatly helped us in improving the present paper. This work is financially supported in part by the Grant-in-Aid of Japanese Ministry of Education, Culture, Sports, Science, and Technology under the contract \#13640245 and \#15540293. 


\section{References}

Allen, S. W., Ettori, S., \& Fabian, A. C. 2001, MNRAS, 324, 877 Arnaud, K. A., Mushotzky, R. F., Ezawa, H., et al. 1994, ApJ, 436, L67

Audit, E., \& Simmons, J. F. L. 1999, MNRAS, 305, L27

Berestetskii, V. B., Lifshitz, E. M., \& Pitaevskii, L. P. 1982, Quantum Electrodynamics (Oxford: Pergamon)

Birkinshaw, M. 1999, Phys. Rep., 310, 97

Buchler, J. R., \& Yueh, W. R. 1976, ApJ, 210, 440

Carlstrom, J. E., Holder, G. P., \& Reese, E. D. 2002, ARA\&A, 40, 643

Challinor, A., Ford, M., \& Lasenby, A. 1999, MNRAS, 312, 159

Challinor, A., \& Lasenby, A. 1998, ApJ, 499, 1

Challinor, A., \& Lasenby, A. 1999, ApJ, 510, 930

David, L. P., Slyz, A., Jones, C., Forman, W., \& Vrtilek, S. D. 1993, ApJ, 412, 479

Dolgov, A. D., Hansen, S. H., Pastor, S., \& Semikoz, D. V. 2001, ApJ, 554,74

Hansen, E., \& Lilje, P. B. 1999, MNRAS, 306, 153

Hansen, S. H. 2003, preprint [astro-ph/0310149]

Holzapfel, W. L., Arnaud, M., Ade, P. A. R., et al. 1997, ApJ, 480, 449

Itoh, N., Kohyama, Y., \& Nozawa, S. 1998, ApJ, 502, 7

Itoh, N., Nozawa, S., \& Kohyama, Y. 2000a, ApJ, 533, 588

Itoh, N., Sakamoto, T., Kusano, S., Nozawa, S., \& Kohyama, Y. 2000b, ApJS, 128, 125

Itoh, N., Kawana, Y., Nozawa, S., \& Kohyama, Y. 2001, MNRAS, 327,567

Itoh, N., Kawana, Y., \& Nozawa, S. 2002a, Nuovo Cimento, 117B, 359

Itoh, N., Sakamoto, T., Kusano, S., Kawana, Y., \& Nozawa, S. 2002b, A\&A, 382, 722
Kitayama, T., Komatsu, E., Ota, N., et al. 2003, PASJ, submitted Kompaneets, A. S. 1957, Sov. Phys. JETP, 4, 730

Markevitch, M. 1998, ApJ, 504, 27

Markevitch, M., Mushotzky, R., Inoue, H., et al. 1996, ApJ, 456, 437

Markevitch, M., Yamashita, K., Furuzawa, A., \& Tawara, Y. 1994, ApJ, 436, L71

Mushotzky, R. F., \& Scharf, C. A. 1997, ApJ, 482, L13

Nozawa, S., Itoh, N., \& Kohyama, Y. 1998a, ApJ, 507, 530

Nozawa, S., Itoh, N., \& Kohyama, Y. 1998b, ApJ, 508, 17

Nozawa, S., Itoh, N., Kawana, Y., \& Kohyama, Y. 2000, ApJ, 536, 31

Rephaeli, Y. 1995, ApJ, 445, 33

Rephaeli, Y., \& Yankovitch, D. 1997, ApJ, 481, L55

Sarazin, C. L. 2003, preprint [astro-ph/0310157]

Sazonov, S. Y., \& Sunyaev, R. A. 1998a, ApJ, 508, 1

Sazonov, S. Y., \& Sunyaev, R. A. 1998b, Astron. Lett., 24, 553

Sazonov, S. Y., \& Sunyaev, R. A. 1999, MNRAS, 310, 765

Schmidt, R. W., Allen, S. W., \& Fabian, A. C. 2001, MNRAS, 327, 1057

Stebbins, A. 1997, preprint [astro-ph/9705178]

Sunyaev, R. A., \& Zeldovich, Ya. B. 1972, Comments Astrophys. Space Sci., 4, 173

Sunyaev, R. A., \& Zeldovich, Ya. B. 1980a, ARA\&A, 18, 537

Sunyaev, R. A., \& Zeldovich, Ya. B. 1980b, MNRAS, 190, 413

Sunyaev, R. A., \& Zeldovich, Ya. B. 1981, Astrophys. Space Phys. Rev., 1,1

Tucker, W., Blanco, P., Rappoport, S., et al. 1998, ApJ, 496, L5

Weymann, R. 1965, Phys. Fluid, 8, 2112

Wright, E. L. 1979, ApJ, 232, 348

Zeldovich, Ya. B., \& Sunyaev, R. A. 1969, Ap\&SS, 4, 301 